\section{Programa Bolsa-Família: qualidade da dieta de população adulta do município de Curitiba, PR}

\section{Bolsa-Família Program: Diet quality of adult population in Curitiba, Paraná}

Flávia Emília Leite de Lima'

Regina Mara Fisberg"

Kátia Yumi Uchimura"I

Telma Picheth"'I

' Departamento de Nutrição da Universidade Federal da Paraíba.

" Departamento de Nutrição, Faculdade de Saúde Pública, Universidade de São

Paulo.

"I' Curso de Nutrição da Faculdade Evangélica do Paraná.

\section{Resumo}

Este estudo avaliou a qualidade da dieta da população beneficiária do Programa BolsaFamília, em Curitiba, PR. Estudo transversal, de base populacional, realizado no período de julho de 2006 a julho de 2007. Foram entrevistados 747 beneficiários, a partir dos 19 anos de idade, de ambos os sexos. Para avaliação da qualidade da dieta foi aplicado recordatório de 24 horas, e o Índice de Qualidade da Dieta (IQD) foi utilizado como parâmetro para classificação do grupo em níveis de consumo. Estatística descritiva foi utilizada para descrever a qualidade da dieta da população. Para a comparação de médias do índice segundo as variáveis socioeconômicas foram realizados o teste $t$ de Wald e a análise de variância ANOVA, considerando-se um nível de significância de $5 \%$. A amostra foi constituída por $91,4 \%$ de mulheres e 8,6\% de homens. A média de idade da população foi de 36,4 \pm 13,3 anos, com cerca de $75 \%$ possuindo o ensino fundamental incompleto. A média do IQD foi de 51 pontos, o que caracteriza uma dieta que precisa de ajustes. A população possui uma dieta monótona, com um consumo adequado de leguminosas, porém baixo para frutas, verduras e produtos lácteos. Na comparação entre as categorias de qualidade da dieta dos indivíduos, todos os componentes, com exceção do sódio, apresentaram medianas de pontuação estatisticamente diferentes $(\mathrm{p}<0,01)$. Estudos que avaliem a qualidade da dieta são fundamentais para subsidiar a implantação de programas de educação alimentar direcionados para o cerne do problema nas populações estudadas.

Palavras-chave: Dieta. Hábitos alimentares. Programas de governo. Índice de qualidade da dieta. Saúde. Alimentos. 


\section{Abstract}

This study evaluated the quality of diet of the population receiving the Bolsa Familia Program in Curitiba, state of Parana, Brazil. It was a population-based cross-sectional study, conducted from July 2006 to July 2007. 747 beneficiaries were interviewed from 19 years of age, of both genders. A 24 hourrecall was implemented in order to assess the quality of the diet and the Healthy Eating Index (HEI) was used as a parameter for the classification of the group in consumption levels. Descriptive statistics were used to describe the diet quality of the studied population. Wald test and ANOVA test were performed to compare the means of the index according to the socio-economic variables, considering a significance level of $5 \%$. The sample comprised $91.4 \%$ of women and $8.6 \%$ ofmen. The average age of the population was $36.4 \pm 13.3$ years, with $75 \%$ having completed elementary school. The mean HEI was 51 points, which features a diet that needs improvement. The population has a monotonous diet with an adequate intake of legumes, but low for fruits, vegetables and dairy products. Comparing the categories of diet quality of individuals, all components, except sodium, showed statistically different median score $(p<0.01)$. Studies that evaluate the quality of the diet are essential to support the implementation of nutrition education programs targeted to the core of the problem in the populations studied.

Keywords: Diet. Food Habits. government programs. Healthy Eating Index. Health. Foods.

\section{Introdução}

As políticas sociais de alimentação e nutrição desenvolvidas no Brasil tem apresentado uma diversidade de propostas, voltadas a equacionar os problemas alimentares e nutricionais da população ${ }^{1}$ .O Bolsa Família, Programa de Transferência Condicionada de Renda (PTCR), tem como objetivo combater a fome, a pobreza e as desigualdades ${ }^{2}$. Sua operacionalização consiste no repasse mensal de benefícios em dinheiro para famílias cadastradas. Segundo Marques et al. ${ }^{3}$, esse programa beneficia cerca de 47 milhões de brasileiros e pode representar até $21 \%$ do orçamento familiar. Attanasio e Mesnard ${ }^{4}$ relatam que no PTCR implementado na Colômbia os recursos recebidos são mais utilizados para a compra de alimentos.

Dados do Instituto Brasileiro de Análises Sociais e Econômicas (IBASE) mostram que os beneficiários do Programa Bolsa-Família (PBF) gastam em média $73 \%$ do benefício com alimentação na Região Sul. O consumo do grupo verduras e legumes nessa região foi o que menos se modificou em relação aos outros após o recebimento do benefício ${ }^{5}$.

A última pesquisa sobre orçamento familiar realizada no Brasil confirma o aumento do consumo de alimentos ricos em gorduras em detrimento àqueles típicos da dieta nacional, como o arroz e o feijão, e de frutas, verduras e legumes. Quando estratificado por renda, observa-se que os feijões, raízes e tubérculos são mais consumidos pela população que tem rendimento de até $1 / 4$ do salário mínimo, cuja dieta é predominantemente glicídica. Quando avaliada a qualidade da alimentação da Região Sul do país observa-se que esta apresenta menor consumo de feijão e maior de gorduras saturadas dentre as cinco regiões ${ }^{6}$.

O PBF caminha na perspectiva de garantir o direito humano à alimentação adequada e, embora não seja um programa de intervenção nutricional, está estreitamente ligado à mudança do estado de saúde $\mathrm{e}$ nutrição da população. As mudanças no consumo de alguns alimentos por essa 
população têm sido demonstradas por meio de estudos, porém é importante identificar para que direção têm seguido os beneficiários em relação à qualidade do que tem sido consumido $^{5,7,8}$.

A abordagem da qualidade da dieta, em termos de alimentos e/ou grupos de alimentos, serve para expressar não só o hábito alimentar, mas também reflete características do contexto sociocultural em que determinada população está inserida ${ }^{9-11}$.

Diante do quadro nutricional ora apresentado no Brasil, e tendo em vista a escassez de estudos com população de tal expressividade, o presente estudo objetiva investigar a qualidade da dieta da população inscrita no Programa Bolsa-Família, no Município de Curitiba, PR.

\section{Casuística e métodos}

Trata-se de um estudo transversal realizado entre os anos de 2006 e 2007, desenvolvido no município de Curitiba, Paraná. Este município está dividido em 9 Distritos Sanitários, cuja demarcação territorial corresponde à divisão do espaço geográfico em administrações regionais.

A população, segundo estimativas do IBGE para 2005, constitui-se de 1.757 .903 habitantes. A taxa de crescimento da cidade vem declinando a cada Censo. O maior crescimento ocorreu entre os anos 70 e 80, quando a taxa registrada foi de $5,34 \%$ ao ano. Considerando o período 1996-2000, a taxa de crescimento anual foi de $1,82 \%^{12}$.

A rede municipal de saúde de Curitiba era composta, em 2005, por 105 Unidades de Saúde (US) e, segundo dados divulgados pelo Ministério do Desenvolvimento Social e Combate à Fome, referentes a 2005, existiam em Curitiba 31.323 famílias inscritas no referido programa naquele $\mathrm{ano}^{13}$.

\section{Seleção da amostra}

Para o cálculo do tamanho da amostra foi considerado o número de famílias inscritas no Programa Bolsa-Família no ano de 2005, residentes na área de adscrição do
Município de Curitiba. O dimensionamento dessa população por distrito sanitário e por unidade de saúde foi disponibilizado pelo setor de Epidemiologia da secretaria municipal de saúde.

O cálculo da amostra considerou a população composta de 31.323 famílias, $3 \%$ de erro tolerável e $5 \%$ de significância, obtendo para este $\mathrm{n}$ amostral um valor de 1.073 domicílios. Os distritos de Bairro Novo e Cajuru foram excluídos da amostra, uma vez que estudo semelhante havia sido realizado no ano anterior à coleta de dados deste estudo, totalizando 747 domicílios $\mathrm{O}$ plano amostral até a escolha do indivíduo a ser entrevistado foi elaborado com base em técnicas de processos probabilísticos, utilizando-se amostra estratificada proporcional, em etapas. Inicialmente considerou-se o distrito sanitário (DS) como estrato, a fim de garantir que, de forma irrestrita, representantes de toda a população beneficiária fossem entrevistados.

Para garantir a representatividade da população no estudo da qualidade da dieta, foram selecionadas famílias adscritas em todas as unidades de saúde do município de Curitiba. Para tanto, após a divisão do $\mathrm{n}$ amostral por distrito sanitário, procedeu-se à distribuição proporcional do número de famílias a serem visitadas, por unidade de saúde.

Para a seleção dos domicílios cujos indivíduos seriam entrevistados, foi realizado um sorteio a partir de uma listagem numerada das famílias.

Definiu-se que estaria apto a participar do estudo o chefe da família ou outro adulto acima de 18 anos, integrante do domicílio, presente no momento da visita. Se por ocasião da visita não houvesse ninguém no domicílio sorteado ou se todos os integrantes do domicílio selecionado se recusassem a participar, o próximo domicílio da lista seria abordado.

\section{Coleta de dados}

A coleta de dados foi desenvolvida por equipes de entrevistadores selecionados 
através de processo seletivo. Participaram das equipes alunos do curso de nutrição e nutricionistas recém-formados. Todos os entrevistadores foram previamente treinados.

A coleta ocorreu simultaneamente em dois distritos sanitários e, para facilitar o acesso dos entrevistadores aos domicílios, contou-se com a colaboração dos agentes comunitários das unidades básicas de saúde.

Os indivíduos foram convidados a participar do estudo, a fim de responder voluntariamente a um questionário sobre hábitos de vida e consumo alimentar.

Foram coletadas informações para descrição do perfil sociodemográfico da população, tais como idade, estado civil, ocupação, número de moradores no domicílio, procedência, escolaridade do entrevistado e do chefe da família.

Para obtenção dos dados sobre o consumo alimentar, foi aplicado um recordatório de 24 horas. Foram registrados todos os alimentos consumidos no dia anterior, com suas respectivas medidas caseiras. Em alguns casos, onde havia dificuldade em dimensionar a medida (ex: fatia pequena de bolo), utilizou-se como recurso auxiliar um álbum de fotografias para inquéritos dietéticos, desenvolvido por Zabotto et al. $(1996)^{14}$. No caso de alguns alimentos para cujo porcionamento não havia fotografia disponível, foi considerado o tamanho da porção referida pelo participante, tomando-se como padrão a tabela de Pinheiro et al. $(2001)^{15}$.

\section{Processamento dos dados}

A composição química dos recordatórios dos indivíduos foi calculada utilizando-se o Programa Virtual Nutri 1.0 ${ }^{16}$. Antes da digitação dos recordatórios no programa procedeu-se à análise crítica dos mesmos a fim de identificar erros na mensuração das quantidades relatadas. Para a digitação de preparações caseiras, tais como pizza, sanduíches, saladas, suas receitas foram desdobradas em seus respectivos ingredientes, a fim de melhor classificar os alimentos segundo os grupos da pirâmide alimentar. Para tanto, foram utilizadas as padronizações de receitas propostas por Pinheiro et al. (2000) ${ }^{16}$ e Fisberg e Villar ${ }^{17}$. Em todas as preparações considerou-se a quantidade de sal de adição separadamente, a fim de verificar de forma mais precisa a quantidade consumida para avaliação do nutriente sódio, componente do IQD. Foram excluídos da amostra os questionários com consumo menor que $500 \mathrm{kcal}$ ou maior que $4000 \mathrm{kcal}$, de acordo com o estabelecido por Willett (1998) ${ }^{18}$.

O consumo alimentar foi avaliado por meio do Índice de Qualidade da Dieta (IQD), um instrumento de medida da qualidade global da alimentação que incorpora nutrientes e alimentos como componentes de qualidade, e estabelece escores de 0 a 10 em cada componente para classificar a alimentação do indivíduo ${ }^{11,19}$.

Indivíduos com uma ingestão igual ao nível recomendado atingem a pontuação máxima de dez pontos. A pontuação mínima de zero é obtida quando nenhum alimento do grupo alimentar foi consumido. Classifica-se como baixo consumo aqueles componentes cuja pontuação é menor que 5 , intermediário de 5 a 8 pontos, e alto acima de 8 pontos.

O valor máximo que o IQD pode atingir é de 100 pontos. Escores altos significam que a ingestão está próxima aos intervalos ou quantidades recomendadas; baixos escores indicam menor conformidade com a recomendação. Foi utilizado no presente estudo o IQD cuja pontuação foi estabelecida por Bowman (1998) ${ }^{20}$ e que classifica os indivíduos com dieta inadequada - até 51 pontos; dieta que necessita de ajustes 51 a 80 pontos; e dieta adequada - mais de 80 pontos.

O processamento de dados para o cálculo do IQD foi realizado por meio de uma série de comandos desenvolvidos em linguagem do software Statistical Package for the Social Sciences for Windows, o qual calcula e atribui a pontuação para cada componente do IQD e para cada indivíduo. 
Utilizou-se estatística descritiva para descrição dos grupos de alimentos. Para verificar a existência de diferenças estatísticas das médias do IQD entre categorias das variáveis sociodemográficas,foi utilizado teste " $t$ " de Student ou análise de variância ANOVA para as variáveis com distribuição normal e mais de duas categorias. Quando encontradas associações estatisticamente significativas foi utilizado o teste de Tukey para identificar as categorias que diferiam entre si.

A fim de verificar a diferença entre as medianas de consumo em cada gupo, de acordo com a pontuação do IQD, utilizou-se o teste de Kruskal-Wallis. Este projeto foi aprovado pelo comitê de ética da Faculdade Evangélica do Paraná e não possui nenhum conflito de interesse entre seus autores.

\section{Resultados}

Foram entrevistados 747 indivíduos. Dentre estes, 91,4\% foram mulheres e 8,6\% homens. A média de idade da população foi de $36,4 \pm 13,3$ anos, com cerca de $75 \%$ possuindo o ensino fundamental incompleto, $35,1 \%$ casados, e $51,7 \%$ tendo como ocupação a função de diarista..

A média do IQD foi de 50,4 pontos. Segundo o índice, $52,9 \%$ foram classificados com dieta inadequada, $45,7,1 \%$ com dieta que necessita de modificações e 1,3\% com dieta adequada.

Verifica-se na Tabela 1 que o IQD apresentou maior média em mulheres, donas de casa, em indivíduos com mais de 40 anos com cônjuge, e possuindo entre 4 e 8

Tabela 1 - Distribuição do número e porcentagem de indivíduos, média do índice de qualidade da dieta (IQD), erro padrão e valor de p segundo variáveis sociodemográficas. Curitiba, 20062007.

Table 1 - Distribution of number and percentage of individuals, health eating index(HEI) means, standard error and " $p$ " value according sociodemographic variables. Curitiba, 2006-2007.

\begin{tabular}{|c|c|c|c|c|c|}
\hline Variável & $\mathrm{N}$ & $\%$ & Média & EP & $p$ \\
\hline Sexo $^{a}$ & & & & & 0,13 \\
\hline Masculino & 63 & 8,4 & 48,02 & 1,62 & \\
\hline Feminino & 683 & 91,6 & 50,62 & 0,51 & \\
\hline Faixa etária (anos) ${ }^{b}$ & & & & & 0,23 \\
\hline$<40$ & 484 & 65,3 & 49,97 & 0,61 & \\
\hline 40 ou mais & 257 & 34,7 & 51,20 & 0,80 & \\
\hline Situação conjugala & & & & & 0,98 \\
\hline Com cônjuge & 450 & 60,3 & 51,26 & 0,62 & \\
\hline Sem cônjuge & 296 & 39,7 & 49,11 & 0,78 & \\
\hline Número de moradores no domicílioc & & & & & 0,38 \\
\hline$<4$ & 480 & 64,5 & 50,10 & 0,60 & \\
\hline 4 ou mais & 263 & 35,5 & 50,99 & 0,83 & \\
\hline Escolaridade (anos de estudo) ${ }^{d}$ & & & & & 0,007 \\
\hline Até 4 anos & 249 & 33,8 & 49,03 & 0,84 & \\
\hline 4 a 8 anos & 313 & 42,5 & 52,26 & 0,78 & \\
\hline$>8$ anos & 175 & 23,7 & 49,33 & 0,88 & \\
\hline $\begin{array}{l}\text { Escolaridade do chefe da família (anos de } \\
\text { estudo) }\end{array}$ & & & & & 0,72 \\
\hline Até 4 anos & 76 & 10,3 & 51,30 & 1,62 & \\
\hline 4 a 8 anos & 552 & 75,0 & 50,49 & 0,56 & \\
\hline$>8$ anos & 108 & 14,7 & 49,72 & 1,17 & \\
\hline Ocupaçãoe & & & & & 0,04 \\
\hline Dona de casa & 385 & 51,7 & 51,38 & 0,69 & \\
\hline Outros & 359 & 48,3 & 49,34 & 0,67 & \\
\hline
\end{tabular}


anos de estudo. As variáveis escolaridades e ocupação mostraram uma diferença de médias estatisticamente significativa entre suas categorias.

A Tabela 2 mostra o consumo médio de energia, gorduras e colesterol segundo categorias do IQD. Observa-se um aumento no consumo de energia à medida que aumentam os escores do índice.

A mediana da pontuação de cada componente do IQD, (Tabela 3), mostra um aumento gradual em todos os grupos alimentares de acordo com as 3 categorias de classificação da qualidade da dieta. Para o colesterol, sódio e percentual de gordura não houve diferença estatisticamente significativa.

A Tabela 4 mostra que o consumo de frutas e produtos lácteos foi muito baixo. $\mathrm{O}$ mesmo se aplica para a variedade da dieta. A mediana de consumo de carnes e feijão foi alta ( $>8$ pontos), porém dois terços da população não consome nenhuma porção de frutas ao dia, $46 \%$ não consomem verduras $\mathrm{e}$ 43,9\% não consome leite e produtos lácteos.

\section{Discussão}

O presente estudo verificou a qualidade da dieta da população beneficiária do Programa Bolsa-Família no Município de Curitiba, que foi demonstrada através do IQD, ferramenta que classifica os indivíduos de acordo com gradientes de ingestão de grupos de alimentos.

A população estudada foi composta basicamente de mulheres e adultos jovens com

Tabela 2 - Consumo médio de energia, gordura total, gordura saturada e colesterol segundo categorias do Índice de Qualidade da Dieta de adultos beneficiários do Programa Bolsa-Família em Curitiba. Curitiba, 2006-2007.

Table 2 - Energy, total fat, saturated fat and cholesterol means intake according to Health Eating Index categories of adults of Bolsa Familia Program in Curitiba. Curitiba, 2006-2007.

\begin{tabular}{lccccc}
\hline IQD escore & $\mathrm{N}$ & $\begin{array}{c}\text { Energia } \\
(\mathrm{kcal})\end{array}$ & $\begin{array}{c}\text { Gord. Total } \\
(\%)\end{array}$ & $\begin{array}{c}\text { Gord Saturada } \\
(\%)\end{array}$ & $\begin{array}{c}\text { Colesterol } \\
(\mathrm{mg})\end{array}$ \\
\hline$<51$ & 395 & 1256,75 & 32,81 & 16,62 & 242,00 \\
$51-80$ & 341 & 1527,43 & 27,51 & 14,43 & 199,70 \\
$>80$ & 11 & 2083,06 & 25,54 & 15,93 & 147,34 \\
\hline
\end{tabular}

Tabela 3 - Valores medianos de escores dos componentes do IQD, segundo a classificação da qualidade da dieta em população adulta beneficiária do programa bolsa família no Município de Curitiba. Curitiba, 2006-2007.

Table 3 - Median values of Diet Quality Index component scores in adult population of Bolsa Família Program in Curitiba. Curitiba, 2006-2007.

\begin{tabular}{|c|c|c|c|c|c|c|}
\hline \multirow{2}{*}{ Componentes } & \multicolumn{2}{|c|}{$\mathrm{IQD}<51^{\mathrm{a}}$} & \multicolumn{2}{|c|}{ IQD $51-80^{a}$} & \multicolumn{2}{|c|}{ IQD $>80^{a}$} \\
\hline & Mediana & DP & Mediana & DP & Mediana & DP \\
\hline Cereais* & 3,71 & 2,49 & 6,30 & 2,73 & 8,50 & 1,80 \\
\hline Verduras* & 0,00 & 1,85 & 1,19 & 3,25 & 10,00 & 3,69 \\
\hline Frutas* & 0,00 & 2,22 & 0,00 & 4,09 & 10,00 & 3,97 \\
\hline Leite* & 0,00 & 1,90 & 1,56 & 2,45 & 4,47 & 3,19 \\
\hline Carnes* & 2,06 & 4,69 & 10,00 & 3,70 & 10,00 & 1,35 \\
\hline Feijão* & 5,72 & 4,38 & 10,00 & 2,94 & 10,00 & 0,22 \\
\hline Colesterol & 10,00 & 3,26 & 10,00 & 2,55 & 10,00 & 0,80 \\
\hline Sódio & 10,00 & 3,82 & 10,00 & 2,15 & 10,00 & 0,81 \\
\hline$\%$ gordura & 10,00 & 3,11 & 10,00 & 2,81 & 10,00 & 2.75 \\
\hline Variedade* & 0,00 & 1,54 & 4,00 & 2,90 & 10,00 & 0,50 \\
\hline
\end{tabular}

$a$ - teste de medianas ${ }^{*} p<0,01 / a-$ test of medians ${ }^{*} p<0.01$ 
Tabela 4 - Estatística Descritiva para os principais componentes de classificação do Índice de Qualidade da Dieta e percentual de indivíduos que obtiveram pontuação mínima ou máxima. Curitiba, 2006-2007.

Table 4 - Descriptive statistics to Diet Quality Index components and porcentual of maximum and minimum score to individuals. Curitiba, 2006-2007.

\begin{tabular}{lccccc}
\hline \multirow{2}{*}{ Componentes } & \multirow{2}{*}{ Média } & \multirow{2}{*}{ Mediana } & \multirow{2}{*}{ Desv. Pad. } & Escore 0 & Escore 10 \\
\hline Cereais & & & & $\%$ & $\%$ \\
Verduras & 5,23 & 4,98 & 2,82 & 2,4 & 10,2 \\
Frutas & 1,86 & $, 4,62$ & 2,83 & 46,0 & 4,7 \\
Leite & 1,87 & 0,00 & 3,63 & 75,3 & 12,2 \\
Carnes & 1,63 & 0,26 & 2,30 & 49,3 & 1,2 \\
Feijão & 6,15 & 9,51 & 4,51 & 30,8 & 48,6 \\
Colesterol & 6,99 & 9,53 & 4,02 & 20,2 & 39,7 \\
Sódio & 7,63 & 10,00 & 3,47 & 8,9 & 54,9 \\
\% gordura & 8,96 & 10,00 & 2,82 & 7,1 & 84,2 \\
Variedade & 8,55 & 10,00 & 2,91 & 5,8 & 69,5 \\
\hline
\end{tabular}

até 8 anos de estudo. Essa caracterização assemelha-se à apresentada no documento síntese do Ibase sobre os beneficiários do Programa Bolsa-Família, que mostra que a maioria dos titulares são mulheres (94\%), possuem entre 15 e 49 anos, são pardos e estudaram somente até $\mathrm{o}$ ensino fundamental (IBASE, 2008).

A média do IQD foi de 51,0 pontos, indicando uma dieta que necessita de modificações, assim como observado em Morimoto et al. (2008) ${ }^{21}$, em estudo desenvolvido com população adulta em municípios da região metropolitana de São Paulo, cuja média do IQD foi de 60,42 pontos.

Estudo realizado com amostra representativa da população norte americana, o Third National Health and Nutrition Examination Survey (NHANES III) demonstrou uma média do IQD de 63,4 pontos ${ }^{20}$. Rafferty et al. (2002) ${ }^{21}$, ao realizarem o estudo The Behavioral Risk Factor Surveillance System (BRFSS), utilizando uma versão modificada do IQD, obtiveram média de 55,7 pontos para pessoas maiores que 18 anos. Champagne et al. (2007) ${ }^{22}$, analisando a qualidade da dieta de população em situação de insegurança alimentar, observaram um IQD de 59,14 para essa população, mostrando que, independente da população em que se avalia a qualidade da dieta, esta tem hábitos que necessitam de modificações.
A escolaridade e a ocupação foram as variáveis que apresentaram diferenças estatisticamente significativas quanto à média do IQD.

A Tabela 2 mostra que à medida que a qualidade da dieta aumenta, aumenta o consumo de energia e diminui o percentual de gordura total e colesterol.

Hoddinott e Skoufias (2003) ${ }^{23}$, em um estudo longitudinal avaliando o impacto do Progresa, programa de transferência de renda do México, no consumo de alimentos, relataram que houve um incremento de calorias e nutrientes nas famílias beneficiárias com aumento da aquisição de vegetais e alimentos de origem animal.

Os resultados do presente estudo corroboram os de Bhattacharya et al. (2004) ${ }^{24}$, que relatam que pessoas mais pobres tendem a possuir escores de IQD mais baixos, porém isso não reflete necessariamente insuficiência de ingestão calórica, mas sim escolhas alimentares inadequadas. A população estudada é caracterizada como pobre ou extremamente pobre, e se observa que a grande maioria está categorizada em uma dieta que necessita de melhoras. Entretanto, a média de consumo de energia não reflete insuficiência calórica para o grupo intermediário e de "dieta adequada" determinados pelo IQD.

Lignani et al. $(2011)^{25}$, ao avaliarem as modificações na alimentação a partir do 
recebimento do Programa Bolsa-Família, com uma amostra de cinco mil domicílios do país, mostraram ter havido aumento em quantidade e variedade do consumo de alimentos, concluindo que o programa aumentou o acesso e ajudou no poder de compra das famílias. Contudo, prevaleceu nessas escolhas uma alimentação de alta densidade calórica e baixo valor nutritivo.

Nigenda e Gonzáles-Robledo (2005) ${ }^{26}$, analisando o impacto dos PTCR latino-americanos, observaram que o Social Protection Network, da Nicarágua, mostrou uma melhoria em relação a qualidade dos alimentos consumidos pelas famílias beneficiárias. No grupo de extrema pobreza, as mudanças foram mais importantes em relação ao tipo de alimento consumido.

O consumo de alguns grupos de alimentos mostrou-se baixo, independente da classificação da qualidade da dieta (Tabela 2). Quase $2 / 3$ da população não referiu nenhum consumo de frutas, e $46 \%$ não consome verduras, corroborando análises de Jaime e Monteiro (2005) ${ }^{27}$ sobre estudo realizado no Brasil, que identifica um consumo diário de frutas menor que $30 \%$ na população brasileira. A pontuação mediana do consumo de verduras foi baixa $(4,62)$, dado este semelhante ao encontrado por Morimoto et al. (2008) ${ }^{10}$.

Lock et al. (2005) ${ }^{28}$, estimando a morbimortalidade atribuível ao baixo consumo de frutas e vegetais, mostraram que este consumo na maioria dos países da América do Sul é menor que no continente africano, em adultos, e que 2,6 milhões de mortes poderiam ser evitadas em todo o mundo, e destas, $31 \%$ de doenças cardiovasculares, caso a população seguisse as recomendações de consumo.

Observando-se os dados da última Pesquisa sobre Orçamentos Familiares (POF) de acordo com rendimento em salários mínimos, a população que possui perfil de usuário de programa de transferência de renda gasta $0,5 \%$ do seu orçamento com frutas e verduras ${ }^{6}$. O baixo consumo de produtos lácteos merece consideração, uma vez que quase $50 \%$ da população apresenta pontuação zero para consumo de leite e derivados, o que demonstra não haver ingestão diária desse grupo.

Estudos realizados nos Estados Unidos e Brasil mostraram um consumo intermediário (entre 5 e 7 pontos) de leite e derivados nas populações estudadas ${ }^{11,21,29}$. Morimoto et al. $(2008)^{10}$, avaliando a dieta em região metropolitana de São Paulo, observaram uma pontuação baixa, assim como no presente estudo, porém apenas $25 \%$ da população estudada apresentou escore zero para esses alimentos. Panigassi et al.(2008) ${ }^{7}$ relataram que famílias em situação de insegurança alimentar consomem menos leite e derivados, verduras, legumes e frutas do que aquelas com situação de segurança.

Levy-Costa et al. $(2005)^{30}$, contrariando os resultados do presente estudo, mostram que a região sul se destaca por possuir maior participação relativa desses alimentos na disponibilidade alimentar do domicílio, inclusive demonstrando que o consumo de leite e derivados vem aumentando com o passar dos anos, mas que em pessoas com faixa de renda até meio salário mínimo a participação relativa de leite e derivados apresentado na última POF representa 3,3\% do total de energia.

O presente estudo tem algumas limitações. Uma delas está na utilização de um recordatório de 24 horas para avaliação da qualidade da dieta. Basiotis et al. (2002) ${ }^{31}$ relatam que os escores calculados a partir de um único dia de recordatório são menores em relação àqueles calculados com 3 dias, porém essa diferença não é significativa. Alguns estudos populacionais que avaliaram a qualidade da dieta também utilizaram apenas um recordatório ${ }^{9,10,26.27}$.

O estudo tem um delineamento transversal, de modo que não foi verificado o consumo anterior ao recebimento do benefício, mas caracterizado o consumo atual, para verificar como têm se alimentado as famílias beneficiárias do programa.

O presente estudo aponta informações fundamentais que fazem emergir a necessidade de trabalhar com essa população, por meio de ações de educação alimentar. 
O Programa Bolsa-Família tem obtido êxito em seus propósitos, porém há que se cuidar dessa população a fim de fomentar melhores escolhas alimentares que podem ser determinantes para saúde. Os resultados ora apresentados mostram-se como subsídio para a construção e implantação de políticas públicas regionais ligadas à educação alimentar e nutricional.

\section{Referências}

1. Uchimura KY, Bosi MLM. Programas de comercialização de alimentos: uma análise das modalidades de intervenção em interface com a cidadania. Rev Nutr Campinas 2003; 16(4): 387-97.

2. BRASIL. Ministério do Desenvolvimento Social e Combate à Fome. Programa Bolsa-Família. 2008. Disponível em: http://www.mds.gov.br. [Acessado em 23 de outubro de 2008]

3. Marques RM, Mendes A. Servindo a dois senhores: as políticas sociais no governo Lula. Rev Katal 2007; 10(1): 15-23.

4. Attanasio O e Mesnard A. The impact of a Conditional Cash Transfer Programme on Consumption in Colombia. Fiscal Studies 2006; 27(4): 421-42.

5. Instituto Brasileiro de Análises Sociais e econômicas (IBASE). Repercussões do programa bolsa família na segurança alimentar e nutricional das famílias beneficiadas. Documento Síntese. 2008.

6. Instituto Brasileiro de Geografia e Estatística - IBGE. Pesquisas de Orçamento Familiar (POF): 2008-2009. Avaliação Nutricional da disponibilidade domiciliar de alimentos no Brasil. Rio de Janeiro; 2010.

7. Panigassi G, Segall-Correa AM, Marin-Leon L et al. Insegurança alimentar intrafamiliar e perfil de consumo de alimentos. Rev Nutr 2008, 21(S): 135-44.

8. Lignani JB e Salles-Costa R. Participação do Programa Bolsa-Família nas modificações do consumo alimentar de famílias beneficiadas pelo programa. Disponível em: http://www.ipc-undp.org/publications/mds/28P.pdf. [Acessado em 18 de janeiro de 2010]

9. Fisberg RM, Marchoni DML, Slater B, Martini LA. Inquéritos Alimentares: Métodos e bases científicos. Ed Atheneu: São Paulo; 2005.

10. Kant AK. Indexes of overall diet quality: a review. J Am Diet Assoc 1996; 96: 785-91.

11. Fisberg RM, Slater B, Barros RR, Lima FD, Carandina L, Barros MBA et al. Índice de qualidade da dieta IQD: avaliação e aplicabilidade. Rev Nutr 2004; 17(4): 301-8.

12. IPPUC. Instituto de Pesquisa e Planejamento Urbano de Curitiba. Área, população e densidade demográfica segundo bairros e regionais de Curitiba 2000-2007. Disponível em: http://ippucnet.ippuc. org.br/Bancodedados/Curitibaemdados/anexos/ ÁreapopulaçãoDensidadeDemográficaSegundo
BairrosRegionaisdeCuritiba.pdf. [Acessado em 28 de novembro de 2007]

13. Morimoto JM, Latorre MRDO, Slater B et al. Dietary Quality and associated factors among adults living in the state of São Paulo, Brazil. J Am Diet Assoc 2006; 106: 2067-72.

14. Zabotto CB. Registro fotográfico para inquéritos dietéticos: utensílios e porções. Campinas, Goiânia: UNICAMP, NEPA; 1996.

15. Pinheiro ABV, Lacerda EMA, Benzecry EH, Gomes MCS, Costa VM. Tabela para avaliação de consumo alimentar em medidas caseiras. 4a Ed. São Paulo: Editora Atheneu; 2000 .

16. Phillippi ST, Szarfarc SC, Latterza AR. Virtual Nutri -Sistema de Análise Nutricional [programa de computador]. Versão 1.0. São Paulo; 1996.

17. Fisberg RM, Villar BS. Manual de Receitas e Medidas Caseiras para Cálculo de Inquéritos Alimentares: manual elaborado para auxiliar o processamento de inquéritos alimentares. São Paulo: Signus Ed.; 2002.

18. Willett WC. Nutritional Epidemiology. 2a ed. New York: Oxford University. 1998.

19. Kennedy ET, Ohls J, Carlson S, Fleming K. The Healthy Eating Index: design and applications. J Am Diet Assoc 1995; 95: 1103-8.

20. Bowman AS, Lino M, Gerrior AS, Basiotis PP. The Healthy Eating Index: 1994-1996. U.S. Department of Agriculture, Center for Nutrition Policy and Promotion;1998.

21. Rafferty AP, Anderson JV, McGee HB, Miller CE. A Healthy Diet Indicator: quantifying compliance with the dietary guidelines using the BRFSS. Prev Med 2002; 35: 9-15.

22. Champagne CM, Casey PH, Connell CL et al. Poverty and Food intake in rural America: Diet Quality is lower in food insecure adults in the Mississipi Delta. J Am Diet Assoc 2007; 107: 1886-94.

23. Hoddinott,J, Skoufias E. The impact of Progress on food consumption. FCND discussion papers 150. Washington D.C.: International Food Policy Research Institute (IFPRI); 2003.

24. Bhattacharya J, Currie J, Haider S. Poverty, food insecurity, and nutritional outcomes in children and adults. J Health Econ 2004; 23(4): 839-62. 
25. Lignani J de B, Sichieri R, Burlandy L, Salles-Costa R. Changes in food consumption among the Programa Bolsa Família participant families in Brazil. Public Health Nutrition 2011; 14(5):785-92.

26. Nigenda G, Gonzáles-Robledo LM. Lessons offered by Latin American cash transfer programmes, Mexico's Oportunidades and Nicaragua's SPN. Implications for African countries. London: DFID Health Systems Resource Centre; 2005.

27. Jaime PC, Monteiro CA. Consumo de frutas e hortaliças na população adulta brasileira, 2003. Cad Saúde Pública 2005: 21(S): 19-24.

28. Locki K, Pomerleau J, Causer L et al. The global burden of disease attributable to low consumption of fruit and vegetables: implications for the global strategy on diet. Bull World Health Organ 2005; 83(2): 100-8.
29. Fisberg RM, Morimoto JM, Slater B, et al. Dietary Quality and Associated Factors among Adults Living in the State of São Paulo, Brazil. J Am Diet Assoc 2006; 106: 2067-72.

30. Levy-Costa RB, Sichieri R, Pontes NS, Monteiro CA. Disponibilidade domiciliar de alimentos no Brasil: distribuição e evolução (1974-2003). Rev Saúde Pública 2005: 39(4): 530-40.

31. Basiotis PP, Carlson A, Gerrior SA, Juan WY, Lino M. The Healthy Eating Index: 1999-2000. U.S. Department of Agriculture, Center for Nutrition Policy and Promotion; 2002.

Recebido em: 02/05/11

Versão final apresentada em: 05/03/12

Aprovado em: 23/05/12 